\title{
Prévision à partir de méthodes chimiques, biologiques et physiques de la digestibilité in vivo et de la teneur en indigestible pariétal de la plante entière de maïs distribuée en vert
}

\author{
J Andrieu, J Aufrère \\ INRA Theix, unité Valeur alimentaire, 63122 Saint-Genès-Champanelle, France
}

\begin{abstract}
Summary - Chemical, biological and physical (NIRS) methods for predicting organic matter digestibility and indigestible cell-wall content of green fodder maize. Relationships between, on the one hand, organic matter digestibility (DMO) or indigestible cell-wall content (NDFI) and, on the other hand, a range of laboratory measurements, have been investigated. These relationships were developed in 99 samples of whole maize plant or corresponding faecal samples. It was found that DMO and NDFI could be more accurately predicted by near infra-red spectroscopy than by faecal cell-wall content, plant acid detergent lignin or by in vitro methods.
\end{abstract}

La digestibilité in vivo chez le mouton de la matière organique $(\mathrm{DMO} \%)$ de la plante entière de maïs est étroitement liée $(R=$ $0,981, E T R=0,66$ ) à sa teneur en indigestible pariétal (NDFI) (Aufrère et al, 1992). Cette étude a pour but de comparer plusieurs méthodes de laboratoire, quant à leur aptitude à prévoir la DMO et le NDFI.

Matériel et méthodes - La DMO de 99 échantillons de mais plante entière "en vert" ( 8 hybrides de maïs dont un hybride BM3 pauvre en lignine) cultivès dans différentes conditions de milieu, a été mesurée sur 4 à 6 moutons alimentés en quantité limitée $(42 \mathrm{~g}$ $M S / \mathrm{kg}$, poids vif 0,75 ) entre le stade laiteux et vitreux. La DMO et la teneur moyenne en NDFI sont respectivement de $72,4 \% \pm 3,1$ et $200 \mathrm{~g} / \mathrm{kg}$ $\mathrm{ms} \pm 30$. Sur la fraction proposée, les teneurs en cendres (MM), matières azotées totales (MAT), cellulose brute (CB), constituants pariétaux (NDF, ADF et ADL, selon la méthode Van Soest), glucides soluble (GLS) et amidon, la digestibilité par la pepsine-cellulase (DCS) (Aufrère et Michalet-Doreau, 1987) et la digestibilité in vitro (DIV) ont été déterminées. La valeur moyenne et l'intervalle de variation de ces différents paramètres sont donnés dans le tableau $I$. La plupart de ces critères (MM, MAT, NDF, $A D F, A D L$ ) ont été également déterminés sur les fèces. Enfin, les spectres d'absorbance (log
$1 / R$ ) ont été mesurés par spectrométrie dans le proche infrarouge (SPIR) sur la fraction proposée et sur les fèces (Norris et al, 1976).

Les relations entre la DMO, le NDFI, d'une part, et l'ensemble des paramètres (chimiques, in vitro et valeurs d'absorbance), d'autre part, ont été établies à l'aide d'un modèle de régression stepwise (SAS, 1985), mais également avec le modèle MPLS pour les seules données d'absorbance (Martens et Naest, 1987).

Résultats et discussion - Pour la fraction proposée, les constituants pariétaux, et particulièrement I'ADL, sont meilleurs prédicteurs de la DMO et du NDFI que les constituants cytoplasmiques (tableau 1). Parmi les méthodes biologiques, la DCS permet une précision supérieure non seulement à celle de la composition chimique, mais aussi à celle de la DIV. L'association de certains critères chimiques à la DIV permet d'améliorer la prévision de la DMO et du NDFI. Enfin, c'est la calibration avec la SPIR (MPLS), qui permet la meilleure prévision de la DMO et du NDFI.

Parmi les critères fécaux, le NDF est le meilleur prédicteur chimique, alors que, curieusement, l'ADL permet une précision inférieure. Sur les fèces, la SPIR (MPLS) 
permet une précision équivalente, voire légèrement supérieure, à celle du NDF.

En conclusion, et en accord avec les résultats de Dardenne et al (1992), la SPIR (MPLS) est la méthode la plus précise, notamment quand elle est réalisée sur la fraction proposée : en effet, elle est supérieure au NDF fécal puis à la DCS et enfin à l'ADL de la fraction proposée et la DIV. Elle permet également de prévoir, avec précision, la composition chimique, la DCS, la DIV (résultats non présentés ici). L'utilisation du modèle stepwise à des fins d'interprétation montre que 2 zones de longueurs d'onde (1 $428 \mathrm{~nm}$ et $1618-1628 \mathrm{~nm}$ ) se retrouvent parmi les plus explicatives, simultanément pour la DMO, le NDFI, les méthodes in vitro, GLS et la lignocellulose.
Aufrère J, Michalet-Doreau B (1987) Anim Feed Sci Technol 20, 203-218

Aufrère J, Graviou D, Demarquilly C, Andrieu J, Émile JC, Giovanni R, Maupetit P (1992) Anim Feed Sci Technol 36, 187-204

Dardenne $P$, Andrieu J, Barrière $Y$, Biston R, Demarquilly C, Femenias N, Lila M, Maupetit $P$, Rivière $F$, Ronsin T (1992) Ann Zootech (sous presse)

Martens H, Naest T (1987) Near Infrared Technology in the Agricultural and Food Industries (P Williams, K Norris, eds) American Association of Cereal Chem Inc, St-Paul, Minnesota, USA, 57-84

Norris KH, Barnes RE, Moore JE, Shenk JS (1976) J Anim Sci 43, 889-897

Statistical Analysis Systems Institute (1985) SAS User's Guide:Basics, Version 4 ed, SAS Institute Inc, Cary, NC

Tableau I. Caractéristiques des échantillons de maïs et prévision de la teneur en parois indigestibles (NDFI) et de la digestibilité de la manière organique (DMO) de la plante entière de maïs à partir de la composition chimique, de méthodes in vitro et de la spectrométrie dans le proche infrarouge (SPIR) mesurées dans la plante ou dans les fèces.

\begin{tabular}{|c|c|c|c|c|c|c|c|c|c|c|}
\hline \multirow{3}{*}{$\begin{array}{l}\text { Variables Carc } \\
\text { explicatives }\end{array}$} & \multicolumn{3}{|c|}{ aractéristiques de la plante } & \multirow{2}{*}{\multicolumn{3}{|c|}{$\begin{array}{l}\text { Teneur en NDFI } \\
\text { de la plante prédite } \\
\text { dartir d'analyses dans : }\end{array}$}} & \multirow{2}{*}{\multicolumn{4}{|c|}{$\begin{array}{c}\text { DMO prédite } \\
\text { à partir d'analyses dans : }\end{array}$}} \\
\hline & \multirow[t]{2}{*}{ Moyenne } & \multirow[t]{2}{*}{ Extrêmes } & à partir d'analyses dans : & & & & & & & \\
\hline & & & \multicolumn{2}{|c|}{ Plante } & \multicolumn{2}{|c|}{ Fèces } & \multicolumn{2}{|c|}{ Plante } & ${ }_{R}^{F \dot{e}}$ & $\begin{array}{l}\text { ces } \\
\text { ETR }\end{array}$ \\
\hline \multirow{8}{*}{$\begin{array}{l}\text { MAT }(\mathrm{g} / \mathrm{kg} \mathrm{ms}) \\
\text { Amidon }(\mathrm{g} / \mathrm{kg} \mathrm{ms}) \\
\text { CB }(\mathrm{g} / \mathrm{kg} \mathrm{ms}) \\
\text { NDF }(\mathrm{g} / \mathrm{kg} \mathrm{ms}) \\
\text { ADF }(\mathrm{g} / \mathrm{kg} \mathrm{ms}) \\
\text { ADL (g/kg ms) } \\
\text { ADL, MAT** } \\
\text { MM, NDF* } \\
\text { DIV (\% } \mathrm{ms}) \\
\text { DCS (\% ms) } \\
\text { MAT, ADL, DIV** }\end{array}$} & b) $\begin{array}{r}77 \\
228\end{array}$ & $\begin{array}{c}52-99 \\
29-388\end{array}$ & $\begin{array}{c}\text { NS } \\
0381\end{array}$ & 276 & 0,775 & 18,9 & NS & 28 & 0,702 & 2,2 \\
\hline & 208 & $157-280$ & 0,598 & 23,9 & $0,892^{*}$ & 13,5 & 0,640 & 2,4 & $0,864^{*}$ & 1,6 \\
\hline & 471 & $389-575$ & 0,568 & 24,6 & $0,927^{*}$ & 11,2 & 0,572 & 2,6 & $0,875^{*}$ & 1,5 \\
\hline & 229 & $174-302$ & 0,649 & 22,7 & 0,900 & 13,0 & 0,670 & 2,3 & 0,832 & 1,7 \\
\hline & 24 & $11-37$ & 0,811 & 17,5 & 0,604 & 23,8 & 0,801 & 1,9 & 0,561 & 2,6 \\
\hline & & & 0,848 & 15 & 0,933 & 10,8 & 0,8 & 1,8 & & \\
\hline & 74,7 & $67,2-85,1$ & 0,755 & 19,6 & & & 0,711 & 2,2 & & \\
\hline & 67,0 & $56,7-77,9$ & $\begin{array}{l}0,863 \\
0,863\end{array}$ & $\begin{array}{l}15,1 \\
15,2\end{array}$ & & & $\begin{array}{l}0,848 \\
0,821\end{array}$ & $\begin{array}{l}1,7 \\
1,8\end{array}$ & & \\
\hline & & & $\mathrm{R}$ & SEC & $R$ & SEC & $R$ & SEC & $\mathrm{R}$ & SEC \\
\hline $\begin{array}{l}\text { SPIR MPLS } \\
\text { SPIR stepwise }\end{array}$ & & & $\begin{array}{l}0,949 \\
0,933\end{array}$ & $\begin{array}{r}9,4 \\
10,8\end{array}$ & $\begin{array}{l}0,933 \\
0,894\end{array}$ & $\begin{array}{l}10,7 \\
13,4\end{array}$ & $\begin{array}{l}0,938 \\
0,900\end{array}$ & $\begin{array}{l}1,1 \\
1,3\end{array}$ & $\begin{array}{l}0,917 \\
0,872\end{array}$ & $\begin{array}{l}1,2 \\
1,5\end{array}$ \\
\hline
\end{tabular}

\footnotetext{
" Modèle curvilinéaire; ${ }^{* *}$ variables significatives $(P<0,01)$ quand les méthodes chimiques et in vitro sont proposées dans le modèle; R : coefficient de corrélation; RSD : écart type résiduel; SEC : écart-type de calibration.
} 